\title{
Point-of-Care Ultrasound Diagnosis of Pneumoperitoneum in the Emergency Department
}

\author{
Melissa Bacci ${ }^{1}$, Roli Kushwaha ${ }^{1}$, Gabriel Cabrera ${ }^{1}$, Eric J. Kalivoda ${ }^{1}$ \\ 1. Emergency Medicine, Hospital Corporation of America West Florida Graduate Medical Education \\ Consortium/Brandon Regional Hospital, University of South Florida Morsani College of Medicine, Brandon, USA
}

Corresponding author: Eric J. Kalivoda, eric.j.kalivoda@gmail.com

\begin{abstract}
Prompt and accurate diagnostic evaluation of the nontraumatic acute abdomen in the emergency department (ED) is crucial to lessen mortality burden. In patients with perforated viscus and pneumoperitoneum, point-of-care ultrasound (POCUS) can assist the emergency physician (EP) in the rapid bedside diagnosis. This report describes a case in which EP-performed POCUS led to the early detection and timely management of an atypical presentation of pneumoperitoneum.
\end{abstract}

Categories: Emergency Medicine

Keywords: point-of-care ultrasound, ultrasonography, pneumoperitoneum, intraperitoneal free air, gastrointestinal perforation, perforated viscus, acute abdomen, emergency department

\section{Introduction}

The critical management of a patient presenting with acute abdominal pain in the emergency department (ED) necessitates rapid diagnostic evaluation, especially given the substantial morbidity- and mortalityassociated sequela of these clinical conditions [1,2]. Gastrointestinal perforation with resultant pneumoperitoneum (intraperitoneal free air secondary to perforated viscus) is an important etiology of acute abdomen cases [3]. Point-of-care ultrasound (POCUS) has a significant and effective role in the prompt bedside diagnosis of many causes of acute abdomen, specifically perforated viscus and pneumoperitoneum $[4,5]$. The sonographic detection of pneumoperitoneum was initially described as the 'Enhanced Peritoneal Stripe Sign (EPSS)', which involves identification of focal hyperechogenic thickening of the peritoneum with associated posterior dirty shadowing and/or horizontal reverberation artifacts [68]. The POCUS findings of pneumoperitoneum have been reported with notably high accuracy; thus, it is an ideal imaging modality for the emergency physician (EP) requiring a timely diagnosis $[9,10]$. Several reports have previously highlighted the value of EP-performed POCUS to diagnose pneumoperitoneum in patients with hemodynamic instability and/or clinical presentations highly suggestive of perforated viscus [1114]. This case report describes the utility of EP-performed POCUS in the early recognition of

Received 05/18/2020

Review began 05/27/2020 Review ended 06/01/2020 Published 06/08/2020

\section{() Copyright 2020}

Bacci et al. This is an open access article distributed under the terms of the Creative Commons Attribution License CC-BY 4.0., which permits unrestricted use, distribution, and reproduction in any medium, provided the original author and source are credited. pneumoperitoneum for the initial evaluation of a patient with an atypical presentation for a life-threatening gastrointestinal perforation.

\section{Case Presentation}

A 52-year-old male with no reported past medical or surgical history presented to the ED with three days of insidious, mild-severity generalized vague abdominal pain with associated nausea, nonbloody nonbilious vomiting, and decreased appetite. He denied fevers, chills, diarrhea, constipation, dark or bloody stools, flank pain, back pain, testicular pain or swelling, genital lesions or discharge, urinary complaints, or any other associated symptoms. Upon arrival, the patient was afebrile, with a blood pressure of 144/102 $\mathrm{mmHg}$, heart rate 114 beats per minute, respiratory rate 16 breaths per minute, and oxygen saturation of $95 \%$ on room air. On physical examination, he was in mild distress and had mild diffuse abdominal tenderness to palpation without rebound, guarding, or peritoneal signs. Of note, examination was unremarkable for scleral icterus, flank or costovertebral angle tenderness, or clinical signs of significant dehydration.

His clinical presentation was relatively nonspecific. However, there was concern for an acute abdominal pathology due to his clinical history and examination. CT imaging of the abdomen/pelvis, laboratory studies, pain medications, intravenous (IV) fluids, blood cultures and broad-spectrum IV antibiotics were ordered. POCUS was subsequently performed by an ultrasound fellowship-trained EP attending and emergency medicine resident physicians. POCUS assessment of the right hypochondrium with a curvilinear transducer in the sagittal plane demonstrated sonographic findings consistent with pneumoperitoneum; focal enhancement of the peritoneal stripe between the liver and abdominal wall and associated reverberation artifacts were appreciated (Figure 1) (Video 1). 


\section{Cureus}

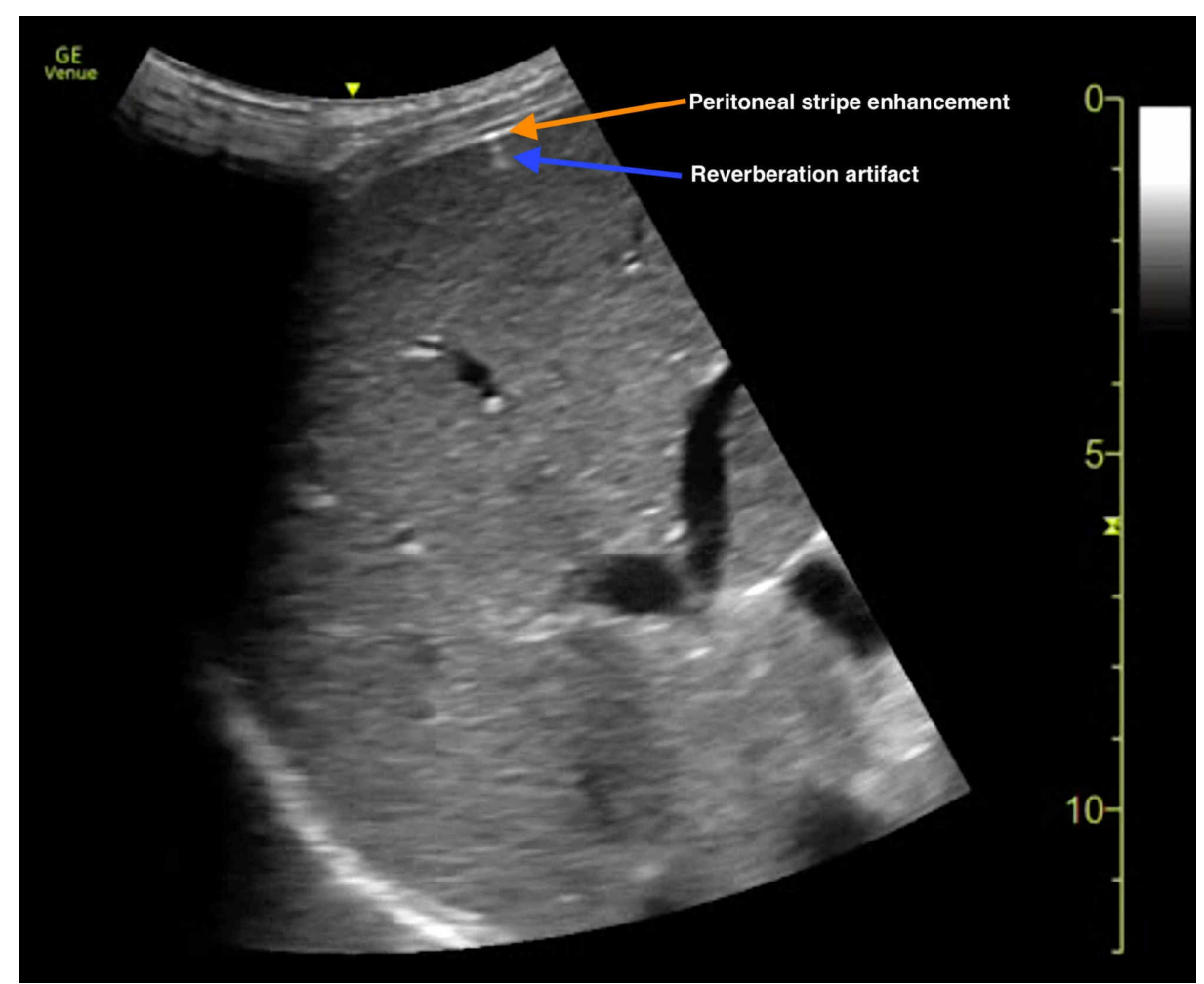

FIGURE 1: Point-of-care ultrasound diagnosis of pneumoperitoneum. Gas bubble abutting the liver and abdominal wall is indicated by peritoneal stripe enhancement (orange arrow) with corresponding reverberation artifact (blue arrow).

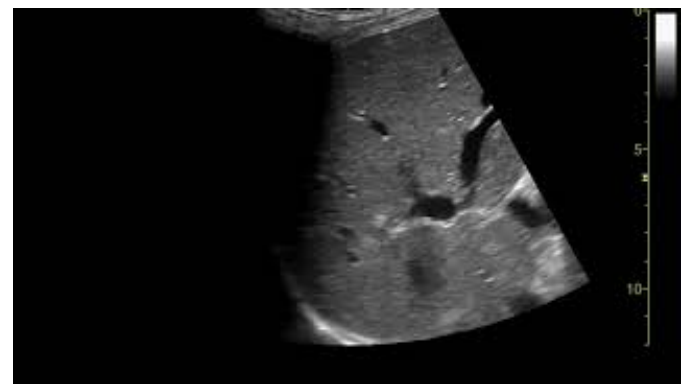

VIDEO 1: Point-of-care ultrasound diagnosis of pneumoperitoneum.

View video here: https://www.youtube.com/watch?v=Pu0ZFVRf3MQ\&feature=youtu.be

POCUS assessment of the bladder demonstrated sonographic evidence of air accumulation, with multiple hyperechoic foci in the anterior bladder wall and associated reverberation artifacts and/or dirty posterior shadowing (Figure 2) (Video 2). Of note, there were no contributing pathologies identified on POCUS evaluation of the gallbladder, kidneys, hepato-renal space, spleno-renal space, or abdominal aorta. 


\section{Cureus}

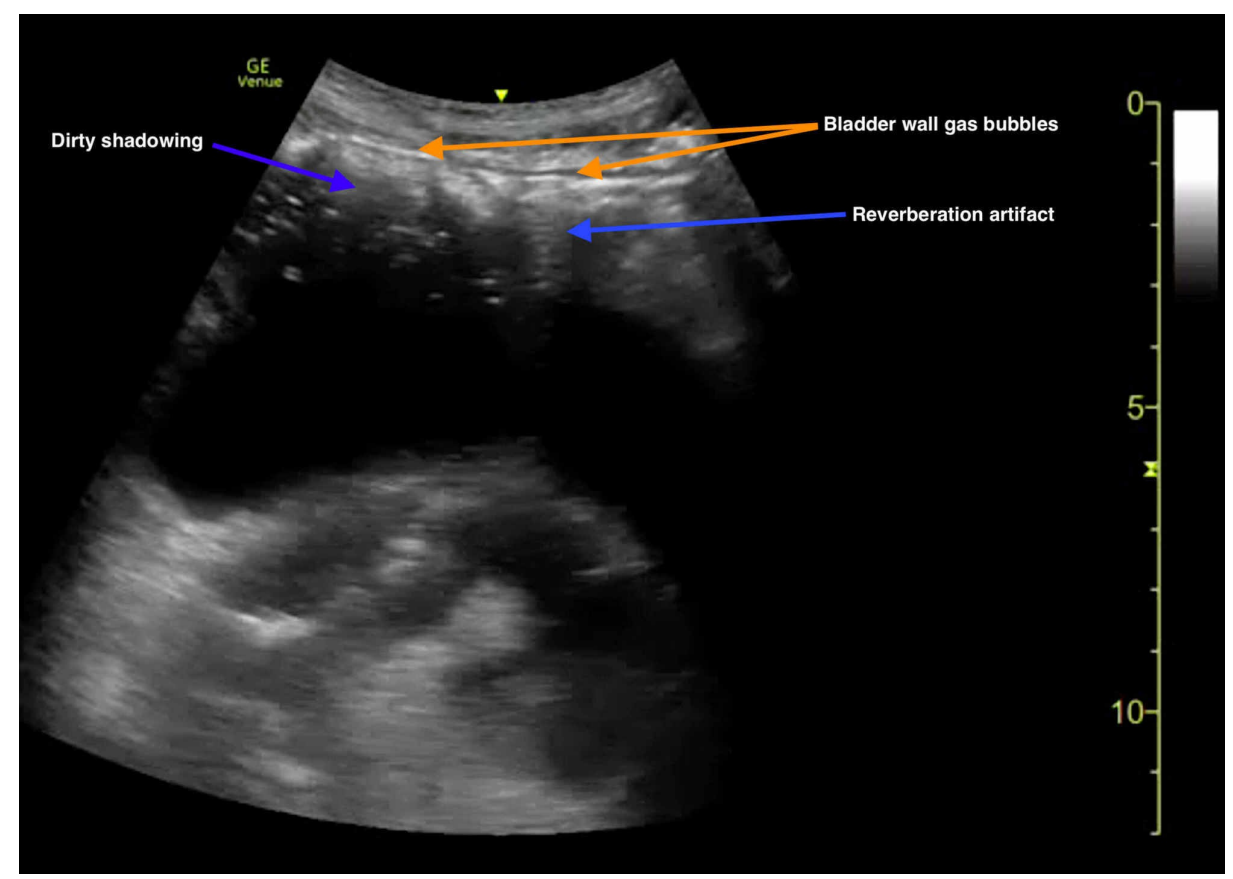

FIGURE 2: Point-of-care ultrasound demonstrating an air-fluid level of the bladder. Gas bubble of anterior bladder wall is indicated by multiple hyperechoic foci (orange arrows) with corresponding reverberation artifact and dirty shadowing (blue arrows).

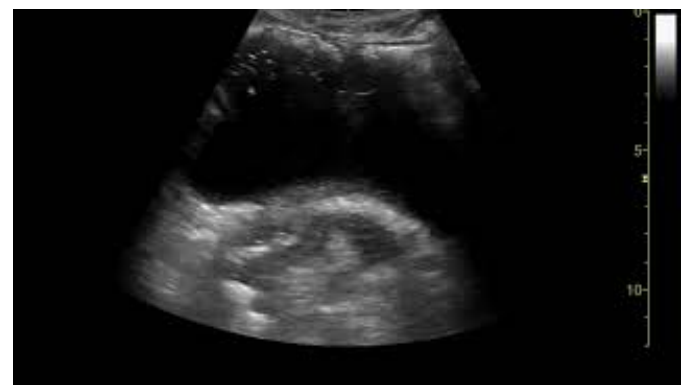

VIDEO 2: Point-of-care ultrasound demonstrating an air-fluid level of the urinary bladder.

View video here: https://www.youtube.com/watch?v=m9i1AKQWedg\&feature=youtu.be

Portable chest radiography was then performed due to the abnormal POCUS findings, which confirmed the presence of intra-abdominal free air on the right consistent with bowel perforation (Figure 3). General surgery was immediately consulted, and surgical preparations were made for emergent exploratory

laparoscopy. Laboratory analysis was notable for white blood cell count of $25.0 \times 10^{3} / \mu \mathrm{L}$ and a venous lactate of $2.0 \mathrm{mmol} / \mathrm{L}$. 


\section{Cureus}

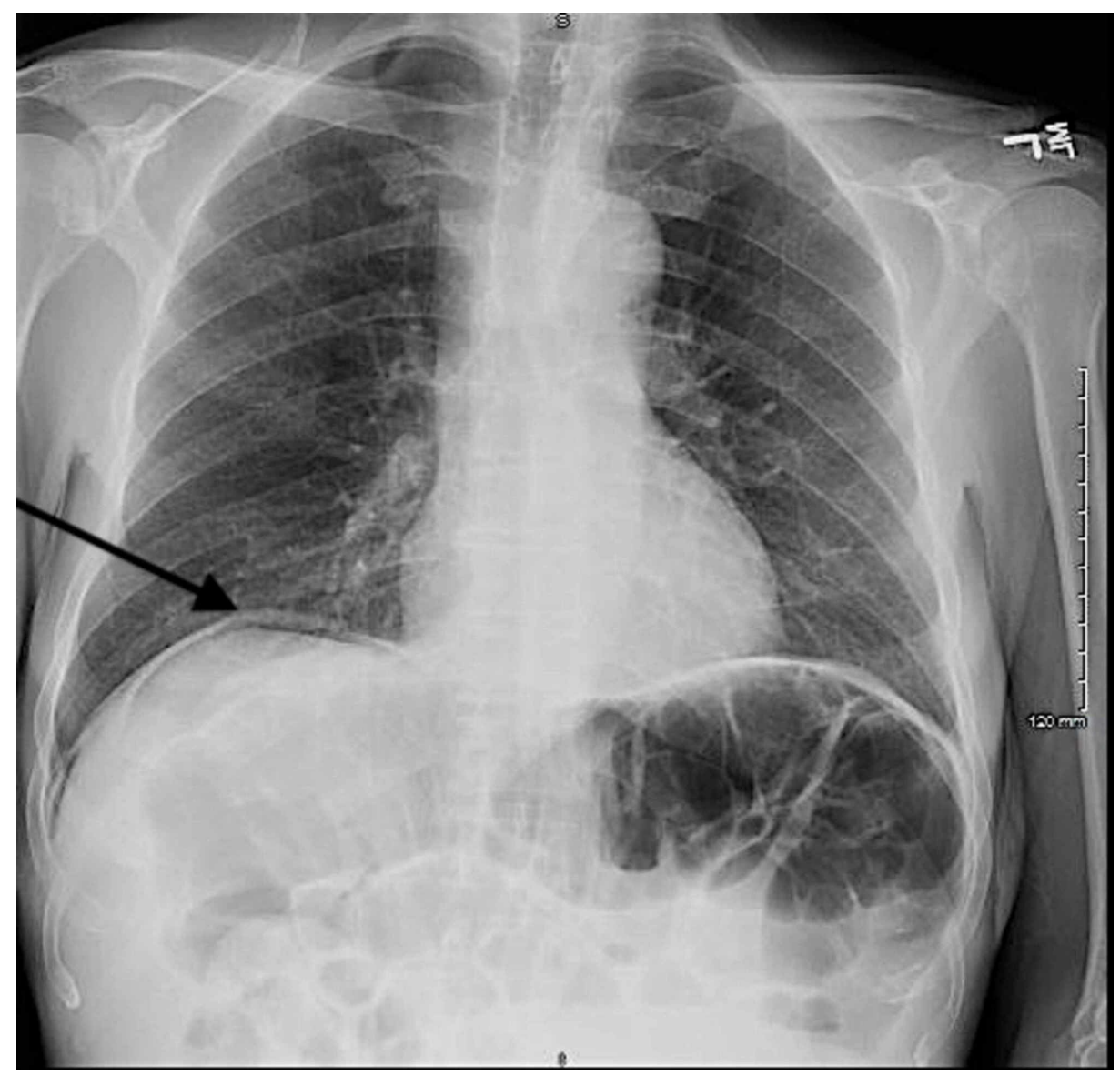

FIGURE 3: Portable chest radiography demonstrating free air under the diaphragm on the right (black arrow) consistent with bowel perforation.

CT of the abdomen/pelvis was obtained en route to the operating room, which ultimately demonstrated intraperitoneal free air, an air-fluid level in the bladder suggesting prior instrumentation or fistula, and sigmoid diverticulitis complicated by multiple abscess collections secondary to sigmoid colon perforation (Figures 4-7). The postoperative surgical report confirmed the diagnoses of perforated sigmoid colon, large pelvic abscesses, and a colovesicular fistula for which a partial colectomy with end colostomy and takedown of colovesicular fistula was performed. The patient was ultimately discharged home in stable condition with instructions for outpatient surgery and urology follow-up. 


\section{Cureus}

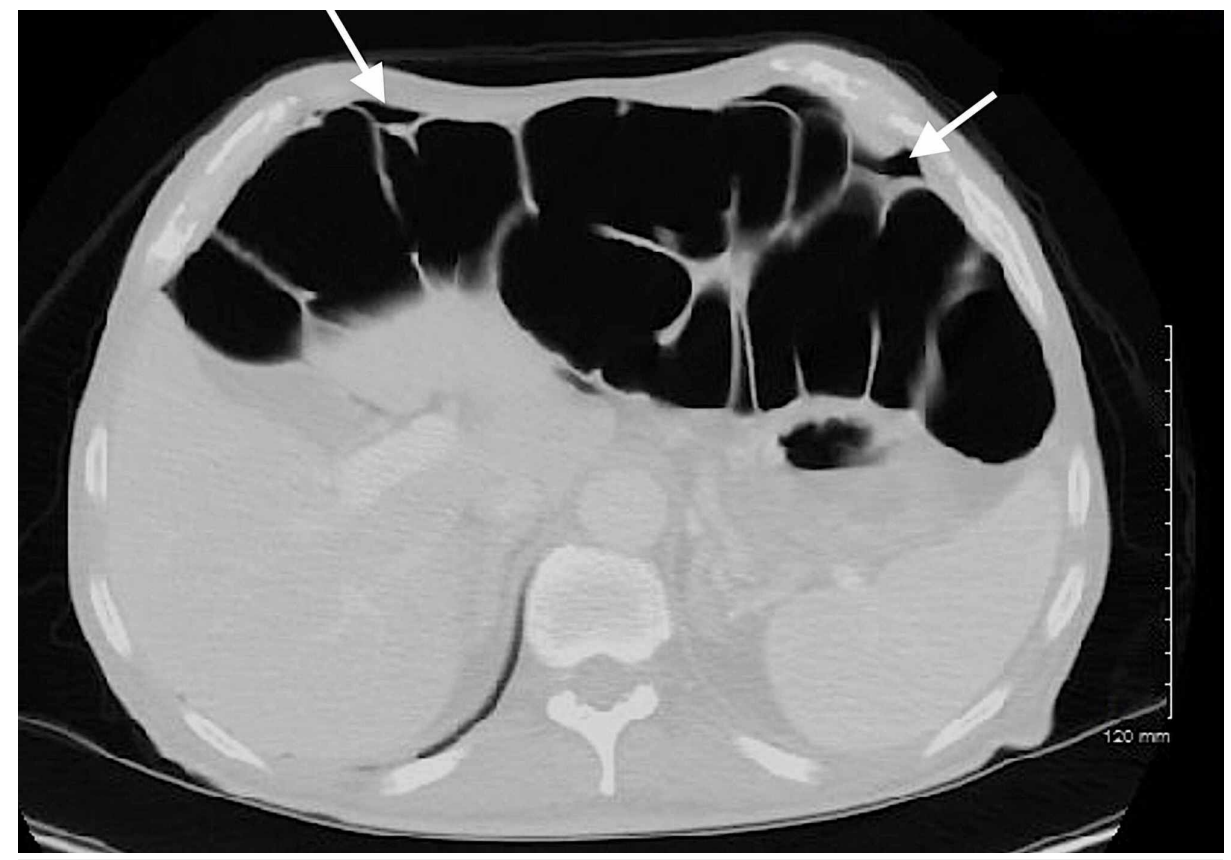

FIGURE 4: CT of the abdomen/pelvis demonstrating intraperitoneal free air (white arrows).

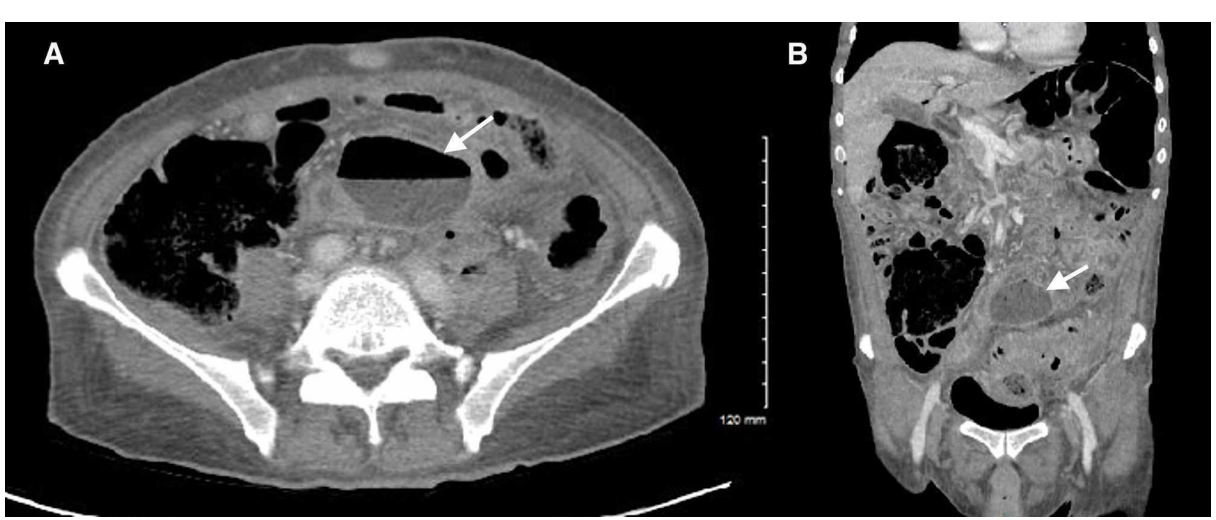

FIGURE 5: CT of the abdomen/pelvis demonstrating an intra-abdominal abscess collection \#1 (white arrows) secondary to sigmoid colon perforation. Axial (A) and coronal (B) planes.

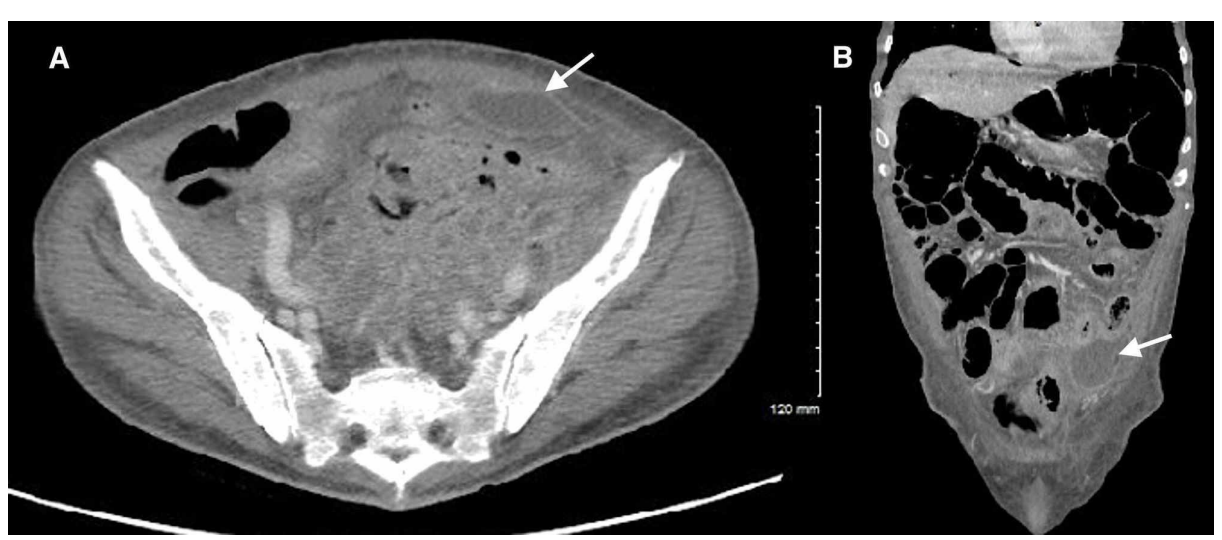

FIGURE 6: CT of the abdomen/pelvis demonstrating an intra-abdominal abscess collection \#2 (white arrows) secondary to sigmoid colon 


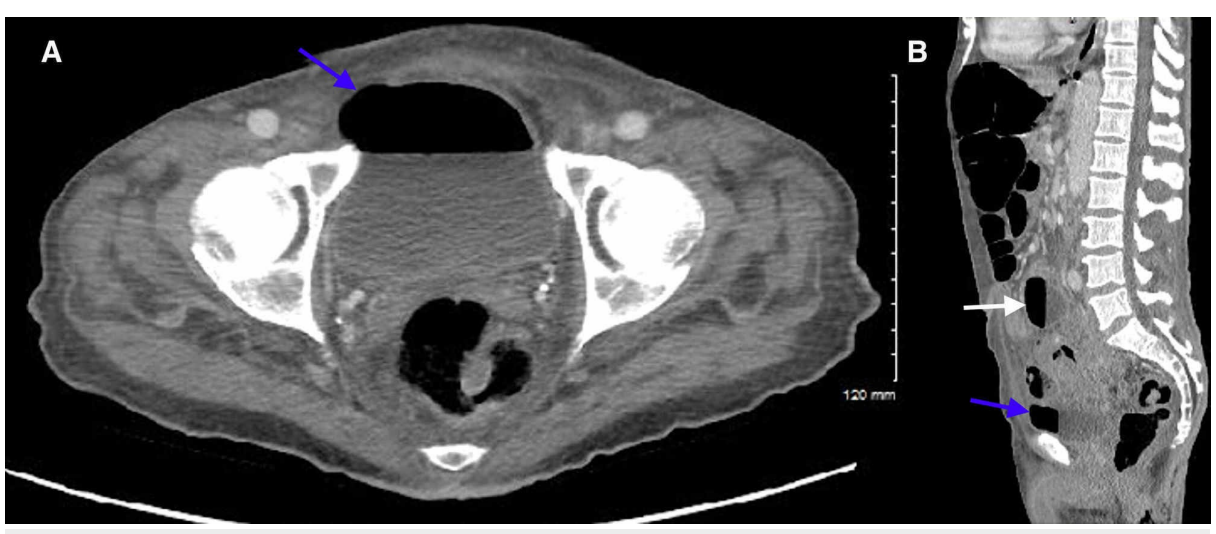

FIGURE 7: CT of the abdomen/pelvis demonstrating air in the urinary bladder (blue arrows) and intra-abdominal abscess collection \#1 (white arrow). Axial (A) and sagittal (B) planes.

\section{Discussion}

Prompt diagnosis can expedite the emergent management of pneumoperitoneum secondary to perforated viscus. EPs can harness the multiple advantages of POCUS in scenarios of acute abdominal pain, chiefly for the rapid primary evaluation and necessary re-assessments depending on the clinical presentation. The conventional practice of initially obtaining plain radiographs for pneumoperitoneum can be of limited diagnostic value in the ED, while multiple studies have highlighted the superior diagnostic sensitivity of ultrasonography compared to plain radiography [15-17]. The diagnostic performance of ultrasonography versus plain radiography for pneumoperitoneum has been reported with sensitivity (93\% versus $79 \%$ ), accuracy ( $90 \%$ versus $77 \%$ ), specificity ( $64 \%$ versus $64 \%$ ), and positive predictive value ( $97 \%$ versus 96\%) [17]. Our patient case demonstrated early bedside sonographic evidence of pneumoperitoneum before any other imaging modalities were obtained, which was significant in that emergent surgical intervention proceeded without delay. CT imaging remains an essential component of the ED diagnostic algorithm in evaluation of perforated viscus, predominantly for its role in downstream surgical planning [18]. There is certainly an emerging role for EP-performed POCUS in the identification of pneumoperitoneum in the ED.

EP training in advanced POCUS for gastrointestinal pathologies is of critical importance [19]. In our opinion, it is imperative that emergency medicine providers are highly skilled in applying the described POCUS techniques for the ED management of suspected cases of pneumoperitoneum. Furthermore, routinely integrating POCUS evaluation of pneumoperitoneum into abdominal-pelvic trauma scenarios has been previously suggested; pneumoperitoneum assessment could be incorporated into the secondary survey as an adjunct to the focused assessment with sonography for trauma (FAST) examination [20]. This case report illustrates the significance of utilizing POCUS in guiding the collaboration of EPs and surgical consultants in the management of perforated viscus.

\section{Conclusions}

Perforated viscus with associated pneumoperitoneum is a life-threatening etiology of acute abdominal pain in the ED. EP-performed POCUS provides an invaluable tool to rapidly diagnose pneumoperitoneum and expedite surgical consultation. Further studies are required to determine the most effective role(s) and timing of POCUS in ED evaluation of pneumoperitoneum in both atraumatic and traumatic clinical situations.

\section{Additional Information \\ Disclosures}

Human subjects: Consent was obtained by all participants in this study. issued approval not applicable. Conflicts of interest: In compliance with the ICMJE uniform disclosure form, all authors declare the following: Payment/services info: All authors have declared that no financial support was received from any organization for the submitted work. Financial relationships: All authors have declared that they have no financial relationships at present or within the previous three years with any organizations that might have an interest in the submitted work. Other relationships: All authors have declared that there are no other relationships or activities that could appear to have influenced the submitted work. 


\section{Acknowledgements}

This research was supported (in whole or in part) by HCA and/or an HCA affiliated entity. The views expressed in this publication represent those of the author(s) and do not necessarily represent the official views of HCA or any other affiliated entities.

\section{References}

1. McNamara R, Dean AJ: Approach to acute abdominal pain. Emerg Med Clin North Am. 2011, 29:159-173. 10.1016/j.emc.2011.01.013

2. Natesan S, Lee J, Volkamer H, Thoureen T: Evidence-based medicine approach to abdominal pain . Emerg Med Clin North Am. 2016, 34:165-190. 10.1016/j.emc.2015.12.008

3. Langell JT, Mulvihill SJ: Gastrointestinal perforation and the acute abdomen. Med Clin North Am. 2008, 92:599-625. 10.1016/j.mcna.2007.12.004

4. Hoffmann B, Nurnberg D, Westergaard MC: Focus on abnormal air: diagnostic ultrasonography for the acute abdomen. Eur J Emerg Med. 2012, 19:284-291. 10.1097/MEJ.0b013e3283543cd3

5. Coppolino F, Gatta G, Di Grezia G, et al.: Gastrointestinal perforation: ultrasonographic diagnosis. Crit Ultrasound J. 2013, 5:S1-S4. 10.1186/2036-7902-5-S1-S4

6. Muradali D, Wilson S, Burns PN, Shapiro H, Hope-Simpson D: A specific sign of pneumoperitoneum on sonography: enhancement of the peritoneal stripe. AJR Am J Roentgenol. 1999, 173:1257-1262. 10.2214/ajr.173.5.10541100

7. Hefny AF, Abu-Zidan FM: Sonographic diagnosis of intraperitoneal free air. J Emerg Trauma Shock. 2011, 4:511-513.

8. Buttar S, Cooper D Jr, Olivieri P, et al.: Air and its sonographic appearance: understanding the artifacts . J Emerg Med. 2017, 53:241-247. 10.1016/j.jemermed.2017.01.054

9. Asrani A: Sonographic diagnosis of pneumoperitoneum using the 'enhancement of the peritoneal stripe sign.' A prospective study. Emerg Radiol. 2007, 14:29-39. 10.1007/s10140-007-0583-3

10. Nazerian P, Tozzetti C, Vanni S, et al.: Accuracy of abdominal ultrasound for the diagnosis of pneumoperitoneum in patients with acute abdominal pain: a pilot study. Crit Ultrasound J. 2015, 7:15. 10.1186/s13089-015-0032-6

11. Jones R: Recognition of pneumoperitoneum using bedside ultrasound in critically ill patients presenting with acute abdominal pain. Am J Emerg Med. 2007, 25:838-841. 10.1016/j.ajem.2007.02.004

12. Blaivas M, Kirkpatrick AW, Rodriguez-Galvez M, Ball CG: Sonographic depiction of intraperitoneal free air. J Trauma. 2009, 67:675. 10.1097/TA.0b013e31804b488d

13. Stone MB, Papanagnou D: Emergency ultrasound identification of pneumoperitoneum. Acad Emerg Med. 2011, 18:30. 10.1111/j.1553-2712.2010.00671.x

14. Chao A, Gharahbaghian L, Perera P: Diagnosis of pneumoperitoneum with bedside ultrasound. West J Emerg Med. 2015, 16:302. 10.5811/westjem.2014.12.24945

15. Chiu YH, Chen JD, Tiu CM, et al.: Reappraisal of radiographic signs of pneumoperitoneum at emergency department. Am J Emerg Med. 2009, 27:320-327. 10.1016/j.ajem.2008.03.004

16. Braccini G, Lamacchia M, Boraschi $P$, et al.: Ultrasound versus plain film in the detection of pneumoperitoneum. Abdom Imaging. 1996, 21:404-412. 10.1007/s002619900092

17. Chen SC, Wang HP, Chen WJ, et al.: Selective use of ultrasonography for the detection of pneumoperitoneum. Acad Emerg Med. 2002, 9:643-645. 10.1111/i.1553-2712.2002.tb02307.x

18. Borofsky S, Taffel M, Khati N, Zeman R, Hill M: The emergency room diagnosis of gastrointestinal tract perforation: the role of CT. Emerg Radiol. 2015, 22:315-327. 10.1007/s10140-014-1283-4

19. Lewiss RE, Pearl M, Nomura JT, et al.: CORD-AEUS: consensus document for the emergency ultrasound milestone project. Acad Emerg Med. 2013, 20:740-745. 10.1111/acem.12164

20. Moriwaki Y, Sugiyama M, Toyoda H, et al.: Ultrasonography for the diagnosis of intraperitoneal free air in chest-abdominal-pelvic blunt trauma and critical acute abdominal pain. Arch Surg. 2009, 144:137-141. 10.1001/archsurg.2008.553 